\title{
Asymptotic behavior of sample paths for retarded stochastic differential equations without dissipativity
}

\section{Zaiming Liu and Na Song ${ }^{*}$}

\author{
"Correspondence: \\ songna@csu.edu.cn \\ School of Mathematics and \\ Statistics, Central South University, \\ Changsha, 410083, China
}

\begin{abstract}
This paper is concerned with two classes of retarded stochastic differential equations. Sufficient conditions are derived to guarantee the pth moment exponential stability and almost sure exponential stability. Moreover, we construct some examples to demonstrate the theory derived.
\end{abstract}

Keywords: retarded stochastic differential equation; variation-of-constants formula; neutral type equation; exponential stability

\section{Introduction}

The theory of retarded stochastic differential equations (SDEs) has received a great deal of attention since it is not only academically challenging but also of practical importance, and it has played an important role in many ways such as in life insurance, risk management, wireless communication, and optimal control of multiagent systems.

Retarded stochastic differential equations (SDEs) are such SDEs that involve retarded arguments. There is extensive literature on the stability of stochastic differential delay equations (see, e.g., [1-7]).

In the past few decades, the theory of neutral stochastic differential equations has also received a great deal of attention. Mao et al. [8] studied the almost sure asymptotic stability of the neutral stochastic differential delay equations with Markovian switching, while Bao et al. [9] investigated the stability in distribution of neutral stochastic differential delay equations with Markovian switching. Wu et al. [10] studied the mean square asymptotic stability of a generalized half-linear neutral stochastic differential equation with variable delays applying fixed point theory. Under a non-Lipschitz condition and a weakened linear growth condition, Bao et al. [11] investigated the existence and uniqueness of mild solutions to stochastic neutral partial functional differential equations. Milošević [12] not only considered global almost sure asymptotic exponential stability of the equilibrium solution for a class of neutral stochastic differential equations with time-dependent delay under nonlinear growth conditions but also established moment estimates for solutions of equations of this type. Bao et al. [13] used a variation-of-constants formula to overcome the difficulties due to the lack of information at the current time and established existence and uniqueness of stationary distributions for retarded SDEs that need not satisfy dissipative conditions. For more details, we refer to [14-17].

(c) 2015 Liu and Song. This article is distributed under the terms of the Creative Commons Attribution 4.0 International License (http://creativecommons.org/licenses/by/4.0/), which permits unrestricted use, distribution, and reproduction in any medium, provided you give appropriate credit to the original author(s) and the source, provide a link to the Creative Commons license, and indicate if changes were made. 
Borrowing the idea of Dung [18], in this paper our investigation is focused on asymptotic behavior of the solution for two classes of retarded SDEs, i.e., semi-linear retarded SDEs and semi-linear retarded SDEs of neutral type. Sufficient conditions are derived to guarantee the $p$ th moment exponential stability and almost sure exponential stability. So far there have been few results presented on the exponential asymptotic behavior of solutions of semi-linear retarded stochastic differential equations. The main aim of this work is to close this gap.

The remainder of this paper proceeds as follows. Section 2 provides some basic notation that will be used in the forthcoming sections and then reviews the variation-of-constants formula for deterministic linear retarded systems. Section 3 is devoted to the study of asymptotic behavior of the solution for semi-linear retarded SDEs and derived sufficient conditions to guarantee the $p$ th moment exponential stability and almost sure exponential stability. Section 4 generalizes the theory established in Section 3 to semi-linear retarded SDEs of neutral type.

\section{Preliminaries}

Let $(\Omega, \mathbb{P}, \mathcal{F})$ be a probability space together with a filtration $\left\{\mathscr{F}_{t}\right\}_{t \geq 0}$ satisfying the usual conditions (i.e., $\mathcal{F}_{t_{+}}:=\bigcap_{s>t} \mathcal{F}_{s}=\mathscr{F}_{t}, \mathscr{F}_{s} \subset \mathscr{F}_{t}$ for $s \leq t$, and $\mathscr{F}_{0}$ contains all $\mathbb{P}$-null sets). Let $\{W(t)\}_{t \geq 0}$ be a real-valued Brownian motion defined on the stochastic basis $\left(\Omega, \mathbb{P}, \mathcal{F},\left\{\mathcal{F}_{t}\right\}_{t \geq 0}\right)$. Fix $\tau \in(0, \infty)$, which is referred to as the delay. $\|\cdot\|_{\text {var }}$ denotes the total variation. Use $\mu(\cdot)$ and $\rho(\cdot)$ to denote the finite signed measures defined on $[-\tau, 0]$. Let $\mathbb{C}$ be the set of all complex numbers and $\operatorname{Re}(z)$ stand for the real part of $z \in \mathbb{C}$. Throughout this paper, $c>0$ is used as a generic positive constant whose values may change for different usage.

Recall the following deterministic linear retarded equation:

$$
d Y(t)=\int_{-\tau}^{0} Y(t+\theta) \mu(d \theta) d t
$$

with the initial value $Y(t)=\xi(t), t \in[-\tau, 0]$.

Define $Z(t)$ as the fundamental solution of equation (1) with the initial value $Z(0)=1$ and $Z(\theta)=0$ for $\theta \in[-\tau, 0]$. By the variation-of-constants formula (see, e.g., [19], Theorem 1.2, p.170), the solution of (1) has the unique explicit form

$$
Y(t ; \xi)=Z(t) \xi(0)+\int_{-\tau}^{0} \int_{\theta}^{0} Z(t+\theta-s) \xi(s) d s \mu(d \theta), \quad t \geq 0
$$

Let

$$
v_{0}=\sup \{\operatorname{Re}(\lambda): \lambda \in \mathbb{C}, \Delta(\lambda)=0\},
$$

where

$$
\Delta(\lambda):=\lambda-\int_{-\tau}^{0} \mathrm{e}^{\lambda s} \mu(d s), \quad \lambda \in \mathbb{C} .
$$


$\Delta(\lambda)=0$ is called the characteristic equation of equation (1) (see, e.g., [19]). Then, according to ([19], Theorem 3.2, p.271), for any $\alpha>v_{0}$, there exists $c_{\alpha}>0$ such that

$$
|Z(t)| \leq c_{\alpha} \mathrm{e}^{\alpha t}, \quad t \geq-\tau
$$

Before the end of this section, we first give the following lemma which will be a crucial tool for proofs of our main results.

Lemma 1 ([18], Lemma 3.1) Suppose that $f \in C\left(\mathbb{R}^{+}, \mathbb{R}^{+}\right) \cap L^{1}\left(\mathbb{R}^{+}\right)$satisfies

$$
\int_{0}^{\infty} f(t) \mathrm{e}^{\gamma t} d t<\infty \text { for some } \gamma>0
$$

If $\lambda>0$ and $\lambda^{\prime}=\lambda \wedge \gamma$, then

$$
\int_{0}^{t} \mathrm{e}^{-\lambda(t-s)} f(s) d s<\mathrm{e}^{-\lambda^{\prime} t} \int_{0}^{\infty} f(s) \mathrm{e}^{\gamma s} d s
$$

\section{Asymptotic behavior of the solution for retarded SDE driven by a Brownian motion}

In this section, we consider a retarded SDE in the form

$$
d X(t)=\left(\int_{-\tau}^{0} X(t+\theta) \mu(d \theta)\right) d t+\sigma(t) d W(t)
$$

with the initial value $X(t)=\xi(t), t \in[-\tau, 0]$.

Theorem 1 ([20], Theorem 3.1) There is a unique strong solution $\{X(t), t \geq 0\}$ of equation (6), and the solution can be represented explicitly by

$$
X(t)=Z(t) \xi(0)+\int_{-\tau}^{0} \int_{\theta}^{0} Z(t+\theta-s) \xi(s) d s \mu(d \theta)+\int_{0}^{t} Z(t-s) \sigma(s) d W(s)
$$

in which $\{Z(t), t \geq-\tau\}$ is the fundamental solution of equation (1) with the initial value $Z(0)=1$ and $Z(\theta)=0$ for $\theta \in[-\tau, 0]$.

The following two theorems provide the $p$ th moment exponential stability and almost sure exponential stability of the solution of equation (6).

Theorem 2 Assume that $v_{0}<0$, and there exists $\gamma>0$ such that

$$
\int_{0}^{\infty} \mathrm{e}^{2 \gamma s}|\sigma(s)|^{2} d s<\infty
$$

Then there exist positive constants $C_{p}$ and $\kappa$ such that, for each $p>0$, the solution of equation (6) satisfies

$$
\mathbb{E}|X(t)|^{p} \leq C_{p} \mathrm{e}^{-\kappa p t}, \quad t \geq 0 .
$$


Proof According to the explicit form (7), along with the following inequality, $\forall x, y, z>0$,

$$
(x+y+z)^{p} \leq c_{p}\left(x^{p}+y^{p}+z^{p}\right)
$$

where $c_{p}=1$ if $0<p \leq 1$ and $c_{p}=3^{p-1}$ if $p>1$, it is easy to get

$$
\begin{aligned}
\mathbb{E}|X(t)|^{p}= & \mathbb{E}\left|Z(t) \xi(0)+\int_{-\tau}^{0} \int_{\theta}^{0} Z(t+\theta-s) \xi(s) d s \mu(d \theta)+\int_{0}^{t} Z(t-s) \sigma(s) d W(s)\right|^{p} \\
\leq & c_{p}\left(\mathbb{E}|Z(t) \xi(0)|^{p}+\mathbb{E}\left|\int_{-\tau}^{0} \int_{\theta}^{0} Z(t+\theta-s) \xi(s) d s \mu(d \theta)\right|^{p}\right. \\
& \left.+\mathbb{E}\left|\int_{0}^{t} Z(t-s) \sigma(s) d W(s)\right|^{p}\right) \\
= & c_{p}\left(I_{1}(t)+I_{2}(t)+I_{3}(t)\right) .
\end{aligned}
$$

We shall consider $I_{i}(t), i=1,2,3$, respectively. For $I_{1}(t)$, by inequality (3), it is obvious that for some $\alpha>0$,

$$
I_{1}(t) \leq c_{\alpha}^{p} \mathrm{e}^{-\alpha p t}\|\xi\|_{\infty}^{p}
$$

For $I_{2}(t)$, by Hölder's inequality and inequality (3),

$$
\begin{aligned}
I_{2}(t) & \leq \mathbb{E}\left|\int_{-\tau}^{0} \int_{\theta}^{0}\right| Z(t+\theta-s)|| \xi(s)\left|d s\|\mu\|_{\mathrm{var}}(d \theta)\right|^{p} \\
& \leq \mathbb{E}\left[\left(\int_{-\tau}^{0}\left(\int_{\theta}^{0}|Z(t+\theta-s)||\xi(s)| d s\right)^{p}\|\mu\|_{\mathrm{var}}(d \theta)\right)^{\frac{1}{p}}\left(\int_{-\tau}^{0}\|\mu\|_{\mathrm{var}}(d \theta)\right)^{\frac{1}{q}}\right]^{p} \\
& =\mathbb{E}\left[\left(\int_{-\tau}^{0}\left(\int_{\theta}^{0}|Z(t+\theta-s)||\xi(s)| d s\right)^{p}\|\mu\|_{\mathrm{var}}(d \theta)\right)\left(\|\mu\|_{\mathrm{var}}([-\tau, 0])\right)^{p-1}\right] \\
& \leq \mathbb{E}\left[\left(\int_{-\tau}^{0} \theta^{p-1} \int_{\theta}^{0}|Z(t+\theta-s)|^{p}|\xi(s)|^{p} d s\|\mu\|_{\mathrm{var}}(d \theta)\right)\left(\|\mu\|_{\mathrm{var}}([-\tau, 0])\right)^{p-1}\right] \\
& \leq c_{\alpha}^{p} \mathrm{e}^{-\alpha p t}\|\xi\|_{\infty}^{p}\left(\|\mu\|_{\mathrm{var}}([-\tau, 0])\right)^{p-1} \int_{-\tau}^{0} \theta^{p-1} \int_{\theta}^{0} \mathrm{e}^{-\alpha p(\theta-s)} d s\|\mu\|_{\mathrm{var}}(d \theta) \\
& \leq(-1 / p)(-\tau)^{p} c_{\alpha}^{p} \mathrm{e}^{\alpha p \tau}\|\xi\|_{\infty}^{p}\left(\|\mu\|_{\mathrm{var}}([-\tau, 0])\right)^{p} \mathrm{e}^{-\alpha p t},
\end{aligned}
$$

where $1 / p+1 / q=1, \forall p, q \in[1, \infty)$.

For $I_{3}(t)$, by Itô isometry formula, we have

$$
\mathbb{E}\left|\int_{0}^{t} Z(t-s) \sigma(s) d W(s)\right|^{2}=\int_{0}^{t}|Z(t-s) \sigma(s)|^{2} d s \leq c_{\alpha}^{2} \int_{0}^{t} \mathrm{e}^{-2 \alpha(t-s)}|\sigma(s)|^{2} d s .
$$

Let $\lambda_{1}=\min (\alpha, \gamma)$ and $\lambda_{2}=\frac{\lambda_{1}}{2}$. By Lemma 1 ,

$$
\mathbb{E}\left|\int_{0}^{t} Z(t-s) \sigma(s) d W(s)\right|^{2} \leq c_{\alpha}^{2} \mathrm{e}^{-2 \lambda_{1} t} \int_{0}^{\infty}|\sigma(s)|^{2} \mathrm{e}^{2 \gamma t} d s=c_{1}^{2} \mathrm{e}^{-2 \lambda_{2} t},
$$

where $c_{1}^{2}:=c_{\alpha}^{2} \mathrm{e}^{-\lambda_{1} t} \int_{0}^{\infty}|\sigma(s)|^{2} \mathrm{e}^{2 \gamma t} d s$ 
Then, by virtue of the Gaussian property, for any $p>0$,

$$
\mathbb{E}|S|^{p}=\frac{2^{p / 2} \Gamma\left(\frac{p+1}{2}\right)}{\Gamma\left(\frac{1}{2}\right)} a^{p}
$$

where $S$ denotes a random variable following an $N\left(0, a^{2}\right)$ law. It is obvious that

$$
I_{3}(t) \leq \frac{2^{p / 2} \Gamma\left(\frac{p+1}{2}\right)}{\Gamma\left(\frac{1}{2}\right)} c_{1}^{p} \mathrm{e}^{-p \lambda_{2} t}
$$

Then, by combining (11), (12) and (16), we conclude that

$$
\begin{aligned}
\mathbb{E}|X(t)|^{p} \leq & c_{p}\left(c_{\alpha}^{p} \mathrm{e}^{-\alpha p t}\|\xi\|_{\infty}^{p}+(-1 / p)(-\tau)^{p} c_{\alpha}^{p} \mathrm{e}^{\alpha p \tau}\|\xi\|_{\infty}^{p}\left(\|\mu\|_{\operatorname{var}}([-\tau, 0])\right)^{p} \mathrm{e}^{-\alpha p t}\right. \\
& \left.+\frac{2^{p / 2} \Gamma\left(\frac{p+1}{2}\right)}{\Gamma\left(\frac{1}{2}\right)} c_{1}^{p} \mathrm{e}^{-p \lambda_{2} t}\right) \\
\leq & C_{p} \mathrm{e}^{-p \kappa t}
\end{aligned}
$$

where $\kappa:=\min \left(\alpha, \lambda_{2}\right)$ and

$$
C_{p}=c_{p}\left(c_{\alpha}^{p}\|\xi\|_{\infty}^{p}+(-1 / p)(-\tau)^{p} c_{\alpha}^{p} \mathrm{e}^{\alpha p \tau}\|\xi\|_{\infty}^{p}\left(\|\mu\|_{\mathrm{var}}([-\tau, 0])\right)^{p}+\frac{2^{p / 2} \Gamma\left(\frac{p+1}{2}\right)}{\Gamma\left(\frac{1}{2}\right)} c_{1}^{p}\right)<\infty
$$

This completes the proof.

Theorem 3 Assume that $v_{0}<0$ and that (8) is satisfied. Then the solution of (6) is almost surely exponentially stable, i.e., there exists $\beta>0$ such that

$$
\limsup _{t \rightarrow \infty} \frac{1}{t} \log |X(t)| \leq-\beta, \quad \text { a.s. }
$$

Proof Note that for $n-1 \leq t \leq n, n \geq 1, X(t)$ can be represented as

$$
X(t)=X(n-1)+\int_{n-1}^{n}\left(\int_{-\tau}^{0} X(s+\theta) \rho(d \theta)\right) d s+\int_{n-1}^{t} \sigma(s) d W(s)
$$

With the inequality $\left|a_{1}+a_{2}+a_{3}\right|^{2} \leq 3\left(\left|a_{1}\right|^{2}+\left|a_{2}\right|^{2}+\left|a_{3}\right|^{2}\right)$, it follows that

$$
\begin{aligned}
\mathbb{E}\left(\sup _{n-1 \leq t \leq n}|X(t)|^{2}\right) \leq & 3\left(\mathbb{E}|X(n-1)|^{2}+\mathbb{E}\left(\int_{n-1}^{n}\left(\int_{-\tau}^{0} X(s+\theta) \rho(d \theta)\right) d s\right)^{2}\right. \\
& \left.+\mathbb{E}\left(\sup _{n-1 \leq t \leq n}\left|\int_{n-1}^{t} \sigma(s) d W(s)\right|^{2}\right)\right) \\
= & 3\left(J_{1}(t)+J_{2}(t)+J_{3}(t)\right) .
\end{aligned}
$$

We shall study $J_{i}(t)$ for $i=1,2,3$, respectively. For $J_{1}(t)$, by inequality (9), it is obvious that

$$
J_{1}(t) \leq C_{2} \mathrm{e}^{-2 \kappa(n-1)}
$$


For $J_{2}(t)$, by Hölder's inequality and inequality (9), we have

$$
\begin{aligned}
J_{2}(t) & \leq \mathbb{E}\left(\int_{n-1}^{n}\left(\int_{-\tau}^{0}|X(s+\theta)|\|\rho\|_{\mathrm{var}}(d \theta)\right) d s\right)^{2} \\
& \leq \mathbb{E}\left[\left(\int_{n-1}^{n} d s\right)^{\frac{1}{2}}\left(\int_{n-1}^{n}\left(\int_{-\tau}^{0}|X(s+\theta)|\|\rho\|_{\mathrm{var}}(d \theta)\right)^{2} d s\right)^{\frac{1}{2}}\right]^{2} \\
& =\mathbb{E}\left(\int_{n-1}^{n}\left(\int_{-\tau}^{0}|X(s+\theta)|\|\rho\|_{\mathrm{var}}(d \theta)\right)^{2} d s\right) \\
& \leq \mathbb{E}\left\{\int_{n-1}^{n}\left[\left(\int_{-\tau}^{0}\|\rho\|_{\mathrm{var}}(d \theta)\right)^{\frac{1}{2}}\left(\int_{-\tau}^{0}|X(s+\theta)|^{2}\|\rho\|_{\mathrm{var}}(d \theta)\right)^{\frac{1}{2}}\right]^{2} d s\right\} \\
& =\|\rho\|_{\mathrm{var}}([-\tau, 0]) \int_{n-1}^{n} \int_{-\tau}^{0} \mathbb{E}^{2}|X(s+\theta)|^{2}\|\rho\|_{\mathrm{var}}(d \theta) d s \\
& \leq\|\rho\|_{\operatorname{var}}([-\tau, 0]) \int_{n-1}^{n} \int_{-\tau}^{0} C_{2} \mathrm{e}^{-2 \kappa(s+\theta)}\|\rho\|_{\mathrm{var}}(d \theta) d s \\
& \leq C_{2}\left(\|\rho\|_{\mathrm{var}}([-\tau, 0])\right)^{2} \mathrm{e}^{2 \kappa \tau} \mathrm{e}^{-2 \kappa(n-1)} .
\end{aligned}
$$

For $J_{3}(t)$, by the Burkholder-Davis-Gundy inequality (see [21], Theorem 7.3, p.40), there exist $c_{2}>0$ and $\lambda_{3}$ such that

$$
J_{3}(t) \leq c_{2} \int_{n-1}^{n}|\sigma(s)|^{2} d s \leq c_{2} \mathrm{e}^{-2 \lambda_{3}(n-1)} \int_{n-1}^{\infty}|\sigma(s)|^{2} \mathrm{e}^{2 \lambda_{3} s} d s \leq c_{3} \mathrm{e}^{-2 \lambda_{3}(n-1)}
$$

where $c_{3}:=c_{2} \int_{0}^{\infty}|\sigma(s)|^{2} \mathrm{e}^{2 \lambda_{3} s} d s<\infty$.

Then, by combining (20)-(22), we conclude that

$$
\begin{aligned}
& \mathbb{E}\left(\sup _{n-1 \leq t \leq n}|X(t)|^{2}\right) \\
& \quad \leq 3\left(C_{2} \mathrm{e}^{-2 \kappa(n-1)}+C_{2}\left(\|\rho\|_{\operatorname{var}}([-\tau, 0])\right)^{2} \mathrm{e}^{2 \kappa \tau} \mathrm{e}^{-2 \kappa(n-1)}+c_{3} \mathrm{e}^{-2 \lambda_{3}(n-1)}\right) \\
& \quad \leq c_{4} \mathrm{e}^{-\lambda_{4} n},
\end{aligned}
$$

where $\lambda_{4}:=\min \left(2 \kappa, 2 \lambda_{3}\right)$ and

$$
c_{4}=3\left(C_{2} \mathrm{e}^{2 \kappa}+C_{2}\left(\|\rho\|_{\mathrm{var}}([-\tau, 0])\right)^{2} \mathrm{e}^{2 \kappa \tau} \mathrm{e}^{2 \kappa}+c_{3} \mathrm{e}^{2 \lambda_{3}}\right) .
$$

Then, with the Chebyshev inequality, for any $\lambda_{5}<\lambda_{4}$,

$$
\mathbb{P}\left(\sup _{n-1 \leq t \leq n}|X(t)|^{2}>\mathrm{e}^{-\lambda_{5} n}\right) \leq \mathrm{e}^{\lambda_{5} n} \mathbb{E}\left(\sup _{n-1 \leq t \leq n}|X(t)|^{2}\right) \leq c_{4} \mathrm{e}^{-\left(\lambda_{4}-\lambda_{5}\right) n}
$$

Since $\sum_{n=1}^{\infty} \mathrm{e}^{-\left(\lambda_{4}-\lambda_{5}\right) n}<\infty$, by virtue of Borel-Cantelli lemma (see [21], Lemma 2.4, p.7), there exists $\Omega_{0} \subset \Omega$ with $P\left(\Omega_{0}\right)=1$ such that for any $\omega \in \Omega_{0}$ there exists an integer $n_{0}(\omega)$, when $n \geq n_{0}(\omega)$ and $n-1 \leq t \leq n$,

$$
|X(t)|^{2} \leq \mathrm{e}^{-\lambda_{5} n} \leq \mathrm{e}^{-\lambda_{5} t}
$$

The desired conclusion (17) is satisfied with $\beta=\frac{\lambda_{5}}{2}$. 
Example 1 Consider the semi-linear retarded SDE

$$
d X(t)=-X(t-1) d t+\sigma(t) d W(t), \quad X(t)=\xi(t)
$$

It is easy to see that the corresponding characteristic equation of (26) is

$$
\lambda+\mathrm{e}^{-\lambda}=0
$$

A simple calculation using Matlab yields that the unique root of (27) is $\lambda=-0.3181+$ 1.3372i. Thus, from this condition, together with (8), we deduce that the solution of (26) is $p$ th moment exponentially stable and almost surely exponentially stable by Theorem 2 and Theorem 3.

\section{Extension to retarded SDE of neutral type}

In this section, we proceed to generalize Theorem 2 and Theorem 3 to SDEs of neutral type. To begin, we give an overview of the variation-of-constants formula for linear equations of neutral type. Recall the following deterministic linear retarded equation of neutral type:

$$
d\left(Y(t)-\int_{-\tau}^{0} Y(t+\theta) \rho(d \theta)\right)=\left(\int_{-\tau}^{0} Y(t+\theta) \mu(d \theta)\right) d t
$$

with the initial value $Y(t)=\xi(t), t \in[-\tau, 0]$.

By ([19], Theorem 1.1, p.256), equation (28) has a unique solution $\{Y(t), t \geq-\tau\}$. Define $Z(t)$ as the fundamental solution of equation (28) with the initial value $Z(0)=1$ and $Z(\theta)=$ 0 for $\theta \in[-\tau, 0]$. Then, for any $\xi \in \mathcal{C}$ such that $\int_{-\tau}^{0}\left|\xi^{\prime}(\theta)\right|^{2} d \theta<\infty, Y(t)$ can be expressed explicitly by

$$
\begin{aligned}
Y(t ; \xi)= & Z(t) \xi(0)-\int_{-\tau}^{0} Z(t+\theta) \xi(0) \rho(d \theta)+\int_{-\tau}^{0} \int_{\theta}^{0} Z(t+\theta-s) \xi(s) d s \mu(d \theta) \\
& +\int_{-\tau}^{0} \int_{\theta}^{0} Z(t-s+\theta) \xi^{\prime}(s) d s \rho(d \theta) .
\end{aligned}
$$

Let

$$
\bar{v}_{0}=\sup \left\{\operatorname{Re}(\lambda): \lambda \in \mathbb{C}, h_{0}(\lambda)=0\right\},
$$

where

$$
h_{0}(\lambda):=\lambda-\lambda \int_{-\tau}^{0} \mathrm{e}^{\lambda \theta} \rho(d \theta)-\int_{-\tau}^{0} \mathrm{e}^{\lambda \theta} \mu(d \theta), \quad \lambda \in \mathbb{C} .
$$

Then, according to ([19], Theorem 3.2, p.271), for any $\bar{\alpha}>\bar{v}_{0}$, there exists $c_{\bar{\alpha}}>0$ such that

$$
|Z(t)| \leq c_{\bar{\alpha}} \mathrm{e}^{\bar{\alpha} t}, \quad t \geq-\tau
$$


In this section, we consider a retarded SDE of neutral type in the form

$$
d\left(X(t)-\int_{-\tau}^{0} X(t+\theta) \rho(d \theta)\right)=\left(\int_{-\tau}^{0} X(t+\theta) \mu(d \theta)\right) d t+\sigma(t) d W(t)
$$

with the initial value $X(t)=\xi(t), t \in[-\tau, 0]$.

Theorem 4 There is a unique strong solution $\{X(t), t \geq 0\}$ of equation (31), and the solution can be represented explicitly by

$$
\begin{aligned}
X(t)= & Z(t) \xi(0)-\int_{-\tau}^{0} Z(t+\theta) \xi(0) \rho(d \theta)+\int_{-\tau}^{0} \int_{\theta}^{0} Z(t+\theta-s) \xi(s) d s \mu(d \theta) \\
& +\int_{-\tau}^{0} \int_{\theta}^{0} Z(t-s+\theta) \xi^{\prime}(s) d s \rho(d \theta)+\int_{0}^{t} Z(t-s) \sigma(s) d W(s),
\end{aligned}
$$

in which $\{Z(t), t \geq-\tau\}$ is the fundamental solution of equation (28) with the initial value $Z(0)=1$ and $Z(\theta)=0$ for $\theta \in[-\tau, 0]$.

The following two theorems provide the $p$ th moment exponential stability and almost sure exponential stability of the solutions to (31).

Theorem 5 Assume that $\bar{v}_{0}<0$ and the following condition holds:

$$
\int_{0}^{\infty} e^{2 \gamma s}|\sigma(s)|^{2} d s<\infty \quad \text { for some } \gamma>0
$$

Then there exist positive constants $\bar{C}_{p}$ and $\bar{\kappa}$ such that, for each $p>0$, the solution of equation (31) satisfies

$$
\mathbb{E}|X(t)|^{p} \leq \bar{C}_{p} \mathrm{e}^{-\bar{\kappa} p t}, \quad t \geq 0
$$

Proof For $\forall a_{i} \in \mathbb{R}, i=1,2, \ldots, n$,

$$
\begin{aligned}
\left(a_{1}+a_{2}+\cdots+a_{n}\right)^{p} & \leq\left(\left|a_{1}\right|+\left|a_{2}\right|+\cdots+\left|a_{n}\right|\right)^{p} \\
& \leq n^{p-1}\left(\left|a_{1}\right|^{p}+\left|a_{2}\right|^{p}+\cdots+\left|a_{n}\right|^{p}\right),
\end{aligned}
$$

we have

$$
\begin{aligned}
\mathbb{E}|X(t)|^{p} \leq & 5^{p-1}\left(\mathbb{E}|Z(t) \xi(0)|^{p}+\mathbb{E}\left|\int_{-\tau}^{0} Z(t+\theta) \xi(0) \rho(d \theta)\right|^{p}\right. \\
& +\mathbb{E}\left|\int_{0}^{t} Z(t-s) \sigma(s) d W(s)\right|^{p}+\mathbb{E}\left|\int_{-\tau}^{0} \int_{\theta}^{0} Z(t+\theta-s) \xi(s) d s \mu(d \theta)\right|^{p} \\
& \left.+\mathbb{E}\left|\int_{-\tau}^{0} \int_{\theta}^{0} Z(t+\theta-s) \xi^{\prime}(s) d s \rho(d \theta)\right|^{p}\right) \\
= & : 5^{p-1}\left(K_{1}(t)+K_{2}(t)+K_{3}(t)+K_{4}(t)+K_{5}(t)\right) .
\end{aligned}
$$


We shall study $K_{i}(t), i=1, \ldots, 5$, respectively. Similarly to the proof of Theorem 2, there exists $\bar{\alpha}>0$ such that

$$
\begin{aligned}
& K_{1}(t) \leq c_{\bar{\alpha}}^{p} \mathrm{e}^{-\bar{\alpha} p t}\|\xi\|_{\infty}^{p}, \\
& K_{3}(t) \leq \frac{2^{p / 2} \Gamma\left(\frac{p+1}{2}\right)}{\Gamma\left(\frac{1}{2}\right)} c_{1}^{p} \mathrm{e}^{-p \lambda_{2} t}
\end{aligned}
$$

and

$$
K_{4}(t) \leq(-1 / p)(-\tau)^{p} c_{\bar{\alpha}}^{p} \mathrm{e}^{\bar{\alpha} p \tau}\|\xi\|_{\infty}^{p}\left(\|\mu\|_{\operatorname{var}}([-\tau, 0])\right)^{p} \mathrm{e}^{-\bar{\alpha} p t}
$$

Then we just need to consider $K_{2}(t)$ and $K_{5}(t)$.

For $K_{2}(t)$, by Hölder's inequality and inequality (30), we have

$$
\begin{aligned}
K_{2}(t) & \leq \mathbb{E}\left|\int_{-\tau}^{0}\right| Z(t+\theta)|| \xi(0)\left|\|\rho\|_{\mathrm{var}}(d \theta)\right|^{p} \\
& \leq \mathbb{E}\left(\left(\|\rho\|_{\mathrm{var}}([-\tau, 0])\right)^{p-1} \int_{-\tau}^{0}|Z(t+\theta)|^{p}|\xi(0)|^{p}\|\rho\|_{\mathrm{var}}(d \theta)\right) \\
& \leq c_{\bar{\alpha}}^{p} \mathrm{e}^{\bar{\alpha} p \tau}\left(\|\rho\|_{\mathrm{var}}([-\tau, 0])\right)^{p} \mathrm{e}^{-\bar{\alpha} p t} .
\end{aligned}
$$

For $K_{5}(t)$, by Hölder's inequality and inequality $(30)$, we get

$$
\begin{aligned}
K_{5}(t) & \leq \mathbb{E}\left|\int_{-\tau}^{0} \int_{\theta}^{0}\right| Z(t+\theta-s)\left\|\xi^{\prime}(s)\left|d s\|\mu\|_{\mathrm{var}}(d \theta)\right|^{p}\right. \\
& \leq \mathbb{E}\left[\left(\int_{-\tau}^{0}\left(\int_{\theta}^{0}\left|Z(t+\theta-s) \| \xi^{\prime}(s)\right| d s\right)^{p}\|\mu\|_{\mathrm{var}}(d \theta)\right)^{\frac{1}{p}}\left(\int_{-\tau}^{0}\|\mu\|_{\mathrm{var}}(d \theta)\right)^{\frac{1}{q}}\right]^{p} \\
& =\mathbb{E}\left[\left(\int_{-\tau}^{0}\left(\int_{\theta}^{0}\left|Z(t+\theta-s) \| \xi^{\prime}(s)\right| d s\right)^{p}\|\mu\|_{\mathrm{var}}(d \theta)\right)\left(\|\mu\|_{\mathrm{var}}([-\tau, 0])\right)^{p-1}\right] \\
& \leq \mathbb{E}\left[\left(\int_{-\tau}^{0} \theta^{p-1} \int_{\theta}^{0}|Z(t+\theta-s)|^{p}\left|\xi^{\prime}(s)\right|^{p} d s\|\mu\|_{\mathrm{var}}(d \theta)\right)\left(\|\mu\|_{\mathrm{var}}([-\tau, 0])\right)^{p-1}\right] \\
& \leq c_{\bar{\alpha}}^{p} \mathrm{e}^{-\bar{\alpha} p t}\left(\|\mu\|_{\mathrm{var}}([-\tau, 0])\right)^{p-1} \int_{-\tau}^{0} \theta^{p-1} \int_{\theta}^{0} \mathrm{e}^{-\bar{\alpha} p(\theta-s)\left|\xi^{\prime}(s)\right|^{p} d s\|\mu\|_{\mathrm{var}}(d \theta) .}
\end{aligned}
$$

Then, by combining (37)-(41), we conclude that

$$
\begin{aligned}
\mathbb{E}|X(t)|^{p} \leq & 5^{p-1}\left(c_{\bar{\alpha}}^{p} \mathrm{e}^{-\bar{\alpha} p t}\|\xi\|_{\infty}^{p}+(-1 / p)(-\tau)^{p} c_{\bar{\alpha}}^{p} \mathrm{e}^{\bar{\alpha} p \tau}\|\xi\|_{\infty}^{p}\left(\|\mu\|_{\text {var }}([-\tau, 0])\right)^{p} \mathrm{e}^{-\bar{\alpha} p t}\right. \\
& +c_{\bar{\alpha}}^{p} \mathrm{e}^{\bar{\alpha} p \tau}\left(\|\rho\|_{\operatorname{var}}([-\tau, 0])\right)^{p} \mathrm{e}^{-\bar{\alpha} p t}+\frac{2^{p / 2} \Gamma\left(\frac{p+1}{2}\right)}{\Gamma\left(\frac{1}{2}\right)} c_{1}^{p} \mathrm{e}^{-p \lambda_{2} t} \\
& \left.+c_{\bar{\alpha}}^{p} \mathrm{e}^{-\bar{\alpha} p t}\left(\|\mu\|_{\operatorname{var}}([-\tau, 0])\right)^{p-1} \int_{-\tau}^{0} \theta^{p-1} \int_{\theta}^{0} \mathrm{e}^{-\bar{\alpha} p(\theta-s)}\left|\xi^{\prime}(s)\right|^{p} d s\|\mu\|_{\operatorname{var}}(d \theta)\right) \\
\leq & \bar{C}_{p} \mathrm{e}^{-p \bar{\kappa} t}
\end{aligned}
$$


where $\kappa:=\min \left(\bar{\alpha}, \lambda_{2}\right)$ and

$$
\begin{aligned}
\bar{C}_{p}= & 5^{p-1}\left(c_{\bar{\alpha}}^{p}\|\xi\|_{\infty}^{p}+(-1 / p)(-\tau)^{p} c_{\bar{\alpha}}^{p} \mathrm{e}^{\bar{\alpha} p \tau}\|\xi\|_{\infty}^{p}\left(\|\mu\|_{\operatorname{var}}([-\tau, 0])\right)^{p}+\frac{2^{p / 2} \Gamma\left(\frac{p+1}{2}\right)}{\Gamma\left(\frac{1}{2}\right)} c_{1}^{p}\right. \\
& +c_{\bar{\alpha}}^{p}\left(\|\mu\|_{\operatorname{var}}([-\tau, 0])\right)^{p-1} \int_{-\tau}^{0} \theta^{p-1} \int_{\theta}^{0} \mathrm{e}^{-\bar{\alpha} p(\theta-s)}\left|\xi^{\prime}(s)\right|^{p} d s\|\mu\|_{\operatorname{var}}(d \theta) \\
& \left.+c_{\bar{\alpha}}^{p} \mathrm{e}^{\bar{\alpha} p \tau}\left(\|\rho\|_{\operatorname{var}}([-\tau, 0])\right)^{p}\right)<\infty .
\end{aligned}
$$

This completes the proof.

Theorem 6 Assume that $\bar{v}_{0}<0$ and that (33) is satisfied. Then the solution of (31) is almost surely exponentially stable, i.e., there exists $\bar{\beta}>0$ such that

$$
\limsup _{t \rightarrow \infty} \frac{1}{t} \log |X(t)| \leq-\bar{\beta}, \quad \text { a.s. }
$$

Proof Note that for $n-1 \leq t \leq n, n \geq 1, X(t)$ can be represented as

$$
\begin{aligned}
X(t)= & X(n-1)+\int_{n-1}^{n}\left(\int_{-\tau}^{0} X(s+\theta) \rho(d \theta)\right) d s+\int_{n-1}^{t} \sigma(s) d W(s) \\
& +\int_{n-1}^{n}\left(\int_{-\tau}^{0} X(s+\theta) \mu(d \theta)\right) d s .
\end{aligned}
$$

With inequality (35), it follows that

$$
\begin{aligned}
\mathbb{E}\left(\sup _{n-1 \leq t \leq n}|X(t)|^{2}\right) \leq & 4\left(\mathbb{E}|X(n-1)|^{2}+\mathbb{E}\left(\int_{n-1}^{n}\left(\int_{-\tau}^{0} X(s+\theta) \rho(d \theta)\right) d s\right)^{2}\right. \\
& +\mathbb{E}\left(\int_{n-1}^{n}\left(\int_{-\tau}^{0} X(s+\theta) \mu(d \theta)\right) d s\right)^{2} \\
& \left.+\mathbb{E}\left(\sup _{n-1 \leq t \leq n}\left|\int_{n-1}^{t} \sigma(s) d W(s)\right|^{2}\right)\right) \\
= & : 4\left(L_{1}(t)+L_{2}(t)+L_{3}(t)+L_{4}(t)\right) .
\end{aligned}
$$

We shall study $L_{i}(t)$ for $i=1,2,3,4$, respectively. For $L_{1}(t)$, by inequality (34), it is obvious that

$$
L_{1}(t) \leq \bar{C}_{2} \mathrm{e}^{-2 \bar{\kappa}(n-1)}
$$

For $L_{2}(t)$, by Hölder's inequality and inequality (34), we have

$$
\begin{aligned}
L_{2}(t) & \leq \mathbb{E}\left(\int_{n-1}^{n}\left(\int_{-\tau}^{0}|X(s+\theta)|\|\rho\|_{\mathrm{var}}(d \theta)\right) d s\right)^{2} \\
& \leq \mathbb{E}\left(\int_{n-1}^{n}\left(\int_{-\tau}^{0}|X(s+\theta)|\|\rho\|_{\mathrm{var}}(d \theta)\right)^{2} d s\right) \\
& \leq \mathbb{E}\left\{\int_{n-1}^{n}\left[\left(\int_{-\tau}^{0}\|\rho\|_{\mathrm{var}}(d \theta)\right)^{\frac{1}{2}}\left(\int_{-\tau}^{0}|X(s+\theta)|^{2}\|\rho\|_{\mathrm{var}}(d \theta)\right)^{\frac{1}{2}}\right]^{2} d s\right\}
\end{aligned}
$$




$$
\begin{aligned}
& =\|\rho\|_{\text {var }}([-\tau, 0]) \int_{n-1}^{n} \int_{-\tau}^{0} \mathbb{E}|X(s+\theta)|^{2}\|\rho\|_{\text {var }}(d \theta) d s \\
& \leq\|\rho\|_{\text {var }}([-\tau, 0]) \int_{n-1}^{n} \int_{-\tau}^{0} \bar{C}_{2} \mathrm{e}^{-2 \bar{\kappa}(s+\theta)}\|\rho\|_{\mathrm{var}}(d \theta) d s \\
& \leq \bar{C}_{2}\left(\|\rho\|_{\mathrm{var}}([-\tau, 0])\right)^{2} \mathrm{e}^{2 \bar{\kappa} \tau} \mathrm{e}^{-2 \kappa(n-1)} .
\end{aligned}
$$

Applying similar techniques to $L_{3}(t)$, we get

$$
L_{3}(t) \leq \bar{C}_{2}\left(\|\mu\|_{\operatorname{var}}([-\tau, 0])\right)^{2} \mathrm{e}^{2 \bar{\kappa} \tau} \mathrm{e}^{-2 \kappa(n-1)} .
$$

For $L_{4}(t)$, by the Burkholder-Davis-Gundy inequality, there exist $c_{2}>0$ and $\lambda_{3}$ such that

$$
L_{4}(t) \leq c_{3} \mathrm{e}^{-2 \lambda_{3}(n-1)}
$$

where $c_{3}:=c_{2} \int_{0}^{\infty}|\sigma(s)|^{2} \mathrm{e}^{2 \lambda_{3} s} d s<\infty$.

Then, by combining (45)-(48), we conclude that

$$
\begin{aligned}
\mathbb{E}\left(\sup _{n-1 \leq t \leq n}|X(t)|^{2}\right) \leq & 4\left(\bar{C}_{2} \mathrm{e}^{-2 \bar{\kappa}(n-1)}+\bar{C}_{2}\left(\|\rho\|_{\mathrm{var}}([-\tau, 0])\right)^{2} \mathrm{e}^{2 \bar{\kappa} \tau} \mathrm{e}^{-2 \kappa(n-1)}\right. \\
& \left.+\bar{C}_{2}\left(\|\mu\|_{\mathrm{var}}([-\tau, 0])\right)^{2} \mathrm{e}^{2 \bar{\kappa} \tau} \mathrm{e}^{-2 \kappa(n-1)}+c_{3} \mathrm{e}^{-2 \lambda_{3}(n-1)}\right) \\
\leq & c_{5} \mathrm{e}^{-\lambda_{6} n}
\end{aligned}
$$

where $\lambda_{6}:=\min \left(2 \bar{\kappa}, 2 \lambda_{3}\right)$ and

$$
c_{5}=4\left(\bar{C}_{2} \mathrm{e}^{2 \bar{\kappa}}+\bar{C}_{2}\left(\|\rho\|_{\operatorname{var}}([-\tau, 0])\right)^{2} \mathrm{e}^{2 \bar{\kappa} \tau} \mathrm{e}^{2 \bar{\kappa}}+\bar{C}_{2}\left(\|\mu\|_{\operatorname{var}}([-\tau, 0])\right)^{2} \mathrm{e}^{2 \bar{\kappa} \tau} \mathrm{e}^{2 \bar{\kappa}}+c_{3} \mathrm{e}^{2 \lambda_{3}}\right) .
$$

Then, in order to carry out arguments analogous to those of (23) and (24), for any $\lambda_{7}<\lambda_{6}$,

$$
|X(t)|^{2} \leq \mathrm{e}^{-\lambda_{7} n} \leq \mathrm{e}^{-\lambda_{7} t}
$$

The desired conclusion (42) is satisfied with $\bar{\beta}=\frac{\lambda_{7}}{2}$.

Example 2 Consider the linear neutral SDE

$$
d\left(X(t)+\frac{1}{3} X(t-1)\right)=-X(t-1) d t+a \int_{-1}^{0} X(t+\theta) d \theta d W(t), \quad X(t)=\xi(t)
$$

where $a \in \mathbb{R}$ and $\{W(t)\}_{t \geq 0}$ is a real-valued Brownian motion defined on the probability space $\left(\Omega, \mathbb{P}, \mathcal{F},\left\{\mathcal{F}_{t}\right\}_{t \geq 0}\right)$.

The characteristic equation associated with the deterministic counterpart of (51) is

$$
\lambda+\left(1+\frac{\lambda}{3}\right) \mathrm{e}^{-\lambda}=0, \quad \lambda \in \mathbb{C}
$$

A calculation by the Matlab shows that the unique root of (52) is $\lambda=-2.313474269$. Thus, from this condition, together with (8), we deduce that the solution of (31) is $p$ th moment exponentially stable and almost surely exponentially stable by Theorem 5 and Theorem 6 . 
Remark 1 In this paper, for notational simplicity, we only treated asymptotic behavior of sample paths for two classes of real-valued retarded SDEs without dissipativity. Our results can be readily generalized to the multidimensional cases. The key is the use of a multidimensional variation-of-constants formula.

\section{Competing interests}

The authors declare that they have no competing interests.

\section{Authors' contributions}

All authors contributed equally to the manuscript. All authors read and approved the final manuscript.

\section{Acknowledgements}

We are very grateful to the anonymous referees and the associate editor for their careful reading and helpful comments.

Received: 20 February 2015 Accepted: 19 May 2015 Published online: 12 June 2015

\section{References}

1. Yorke, JA: Asymptotic stability for one dimensional differential-delay equations. J. Differ. Equ. 7(1), 189-202 (1970)

2. Mao, X: Exponential stability of equidistant Euler-Maruyama approximations of stochastic differential delay equations. J. Comput. Appl. Math. 200(1), 297-316 (2007)

3. Hou, Z, Bao, J, Yuan, C: Exponential stability of energy solutions to stochastic partial differential equations with variable delays and jumps. J. Math. Anal. Appl. 366(1), 44-54 (2010)

4. Scheutzow, M: Exponential growth rate for a singular linear stochastic delay differential equation. arXiv:1201.2599 (2012)

5. Bao, J, Yin, G, Wang, L, Yuan, C: Exponential mixing for retarded stochastic differential equations. arXiv:1306.3585 (2013)

6. Bao, J, Yin, G, Yuan, C: Exponential ergodicity for retarded stochastic differential equations. Appl. Anal. 93(11), 2330-2349 (2014)

7. Bao, J, Hou, Z, Yuan, C: Stability in distribution of mild solutions to stochastic partial differential equations. Proc. Am. Math. Soc. 138(6), 2169-2180 (2010)

8. Mao, $X$, Shen, Y, Yuan, C: Almost surely asymptotic stability of neutral stochastic differential delay equations with Markovian switching. Stoch. Process. Appl. 118(8), 1385-1406 (2008)

9. Bao, J, Hou, Z, Yuan, C: Stability in distribution of neutral stochastic differential delay equations with Markovian switching. Stat. Probab. Lett. 79(15), 1663-1673 (2009)

10. Wu, M, Huang, NJ, Zhao, CW: Stability of half-linear neutral stochastic differential equations with delays. Bull. Aust. Math. Soc. 80(3), 369-383 (2009)

11. Bao, J, Hou, Z: Existence of mild solutions to stochastic neutral partial functional differential equations with non-Lipschitz coefficients. Comput. Math. Appl. 59(1), 207-214 (2010)

12. Milosevic, M: Almost sure exponential stability of solutions to highly nonlinear neutral stochastic differential equations with time-dependent delay and the Euler-Maruyama approximation. Math. Comput. Model. 57(3), 887-899 (2013)

13. Bao, J, Yin, G, Yuan, C: Stationary distributions for retarded stochastic differential equations without dissipativity. arXiv:1308.2018 (2013)

14. Bao, J, Hou, Z, Wang, F: Exponential stability in mean square of impulsive stochastic difference equations with continuous time. Appl. Math. Lett. 22(5), 749-753 (2009)

15. Bao, J, Truman, A, Yuan, C: Almost sure asymptotic stability of stochastic partial differential equations with jumps. SIAM J. Control Optim. 49(2), 771-787 (2011)

16. Bao, J, Yuan, C: Numerical approximation of stationary distributions for stochastic partial differential equations. J. Appl. Probab. 51(3), 858-873 (2014)

17. Bao, J, Yuan, C: Large deviations for neutral functional SDEs with jumps. Stochastics 87(1), 48-70 (2015)

18. Dung, N: Asymptotic behavior of linear fractional stochastic differential equations with time-varying delays. Commun. Nonlinear Sci. Numer. Simul. 19(1), 1-7 (2014)

19. Hale, JK: Introduction to Functional Differential Equations. Springer, New York (1993)

20. Reiß, M, Riedle, M, Gaans, OV: Delay differential equations driven by Lévy processes: stationarity and Feller properties. Stoch. Process. Appl. 116(10), 1409-1432 (2006)

21. Mao, X: Stochastic Differential Equations and Applications. Horwood, Chichester (2007) 